(C) IEEE

https://doi.org/10.1109/dsd.2019.00066

Castillejo, Pedro, Baran Curuklu, Roberto Fresco, Gor m Johansen, Sonia Bilbao-Arechabala, Belen Martinez-R odriguez, Luigi Pomante, Jose-Fernan Martinez-Ortega, and Marco Santic. "The AFarCloud ECSEL Project." 2019 22nd Euromicro Conference on Digital System Design (D SD) (August 2019). doi:10.1109/dsd.2019.00066. 


\section{The AFarCloud ECSEL Project}

\author{
Pedro Castillejo \\ Universidad Politecnica de Madrid \\ Madrid, SPAIN \\ pedro.castillejo@upm.es \\ Gorm Johansen \\ SINTEF \\ Trondheim, Norway \\ gorm.johansen@sintef.no \\ Luigi Pomante \\ Università degli Studi dell’Aquila \\ Center of Excellence DEWS \\ L'Aquila, ITALY \\ luigi.pomante@univaq.it
}

\author{
Baran Cürüklü \\ Mälardalen University \\ Västerås, Sweden \\ baran.curuklu@mdh.se \\ Bilbao Arechabala Sonia \\ Tecnalia \\ Bizkaia, SPAIN \\ sonia.bilbao@tecnalia.com \\ José-Fernán Martínez-Ortega \\ Universidad Politecnica de Madrid \\ Madrid, SPAIN \\ jf.martinez@upm.es
}

\author{
Roberto Fresco \\ CNR - IMAMOTER \\ Ferrara, ITALY \\ r.fresco@imamoter.cnr.it \\ Belen Martinez \\ Tecnalia \\ Bizkaia, SPAIN \\ belen.martinez@tecnalia.com \\ Marco Santic \\ Università degli Studi dell'Aquila \\ Center of Excellence DEWS \\ L'Aquila, ITALY \\ marco.santic@univaq.it
}

\begin{abstract}
Farming is facing many economic challenges in terms of productivity and cost-effectiveness. Labor shortage partly due to depopulation of rural areas, especially in Europe, is another challenge. Domain specific problems such as accurate identification and proper quantification of pathogens affecting plant and animal health are key factors for minimizing economical risks, and not risking human health. The ECSEL AFarCloud (Aggregate FARming in the CLOUD) project will provide a distributed platform for autonomous farming that will allow the integration and cooperation of agriculture Cyber Physical Systems in real-time in order to increase efficiency, productivity, animal health, food quality and reduce farm labour costs. This platform will be integrated with farm management software and will support monitoring and decision-making solutions based on big data and real time data mining techniques.
\end{abstract}

Keywords-Cyber-Physical Systems, Smart \& Precision Farming, Livestock management, crop monitoring, autonomy and cooperation, autonomous and semi-autonomous vehicles, farming robots

\section{INTRODUCTION}

Modern agriculture is facing tremendous challenges in order to build a sustainable future across different regions of the globe. Examples of such global challenges include population increase, urbanization, an increasingly degraded environment, an increasing trend towards consumption of animal protein, changing in food preferences trough aging population and migration, and of course climate change [1]. Global efforts will need to be addressed in a way that does not endanger the capacity of the agriculture sectors - crops, livestock, fisheries and forestry - to meet the world's food needs.

Precision farming and automation have already established paradigms in order to increase farm productivity, quality, as well as improving working conditions through reducing manual labour. All these factors play an important role in helping to make farming become sustainable. Also, many modern farmers already use high-tech solutions, e.g. digitally-controlled farm implements, and even unmanned aerial vehicles for monitoring and forecasting. There are partially and fully automatic devices for most aspects of agricultural functions from grafting to seeding and planting, from harvesting to sorting, packaging and boxing, and livestock management. The drawbacks of these approaches, even though they can be effective and useful for farmers, is that these systems are calibrated only for a specific task, without the ability to provide a holistic solutions and view of agricultural processes. In addition, there is a lack of interoperability causes additional work for the users, as they have to manually feed the output data from one system into the next. The combination of specific solutions and lack of interoperability is preventing the uptake of the advanced solutions. By integrating the different parts of a whole infrastructure, such as software modules, vehicles/UAVs and other equipment through a middleware with a common information model and application interface, AFarCloud (Aggregate FARming in the CLOUD) will allow a reduction in monotonous, and time consuming work, in addition to opening up data, which is already available, to new uses and applications.

In particular, it will be possible to face other challenges in environmental impact, public health and energy. In fact, although an important part of human society, agriculture has several negative impacts on the environment. It adds globally significant and environmentally detrimental amounts of nitrogen and phosphorus to terrestrial ecosystems [2][3]. In addition to the environmental impact, there is a health aspect to the use of chemicals in agriculture. These chemicals present an occupational threat extending to farm workers, their families and potentially to inhabitants of areas exposed to their application on crops, on vineyards etc. Index of risk of damage from pesticide toxicity and exposure can be determined [4][5] and, through autonomous precision farming, these effects can be mitigated as chemicals such as fertilizer and pesticides are only administered where needed instead of applied over a large area. Lastly, there are several aspects of precision farming that will reduce energy consumption in farming. One way of doing this is using multiple light autonomous vehicles instead of a few heavy tractors, soil compaction is reduced, which again reduces the energy needed for tilling the soil. Additionally, $\mathrm{CO}_{2}-$ emissions will be reduced as the use of electric ground vehicles and UAVs partially replace large fossil fuel-based vehicles.

The AFarCloud project has started on September 1st, 2018 and its duration is three years. In the following, we highlight project goals, explain selected approach, describe application domains, and discuss main implementation issues. 


\section{THE AFARCLOUD SOLUTION}

As reported in the Scientific and Technological Options Assessment (STOA) Study of European Parliament, precision farming technologies allow the production of 'more with less'. The use of natural resources, agrochemicals, antibiotics and energy will be reduced to the benefit of both farmers and the environment, and in turn society, with the above described factors. AFarCloud proposal assumes that, in order to address these inter-related challenges, there is an urgent need for a holistic and systematic approach, through smart sustainable and digital automated production. It needs to consider different application capabilities like the data collection and cloud computing, a sensing-on-the-move approach, cyber physical systems (CPS), IoT actuation, decision support systems, autonomous vehicles like UAVs and other partially and fully automatic devices for most aspects of agricultural processes, with the objective of realizing farming-as-a-service, adding a real-time monitoring level in a farm viewed as a whole. In other terms, a novel holistic view of farming and of everything tightly connected with the optimization of production, energy, food quality and services, in order to promote a healthier life-style and reduced labour levels for workers.

The AFarCloud project will promote novel precision farming solutions by providing cyber physical systems (CPS), as well as a monitoring and sensing framework able to utilize new autonomous robotics platforms and incorporating the legacy systems already deployed in the farms. Services and technologies developed in the AFarCloud project will result in resource-efficient and environmentally friendly agriculture, also leading to a supply chain approach with food security and quality as its main pillars. In addition, the proposed solution will provide detection, accurate identification and proper quantification of factors affecting plants and animal health. Moreover, this project will deliver a reference open software architecture providing a comprehensive approach to a "farming-as-aservice" and a novel business solution, considering affordable modular solutions and integration with preexisting machinery in farms. The farmers will benefit from selective harvesting, reduced exposure risks, targeted weed reduction and fertilization, livestock surveillance, automated livestock management and planning. AFarCloud will include a surveillance framework for crop/livestock. By analysing and processing images and/or videos, the platform will be able to predict or recognize situations that could damage the crop and livestock security, as for example, the tracking of livestock inside a defined perimeter.

The AFarCloud project will be executed with a set of demonstrator's sites in order to provide significant application scenarios for the whole platform during the timeframes of the project. Crop and livestock sites for the scenarios will offer the possibility to analyse better quality life-style in farmers and their workload, with a consistent reduction in pollution, agrochemicals and pesticide exposures also in the surrounding areas of the demonstrator site. This objective will be assessed by environmental monitoring and epidemiological surveys.

The AFarCloud project will contribute to:

- Enhance the applications of Cyber-Physical Systems in the farming domain.
- Improve the autonomy and cooperation of farming CPS solutions.

- Increase the interoperability, cooperation, and reuse of CPS and autonomous vehicles achieving a better level of reduction of human labour.

- Enable reliable, high-performance, real-time and secure data exchange for CPS.

- Environmental benefits and reduction of agrochemicals in farming activities, contribution to biodiversity protection as well, by adopting ecofriendly agronomic protocols that AFarCloud technology will support in order to calibrate them effectively in real time monitoring.

- Optimization of resources used in agriculture and better approach for pesticide-free food production. Benefits for farm workers and people living in surrounding areas of the farm will be achieved, related to the reduction of agrochemicals.

- Generate a direct impact and innovation in the EU farming and mechatronics industry, by providing new standards in the UAV and UGV industry, in order to demonstrate and apply a structured and cost-effective approach to the development of new farming solutions.

Some concrete services the system will provide to farmers and other stakeholders are:

- Real time data access, combined with big data and data mining techniques for monitoring physiological parameters.

- Auto steering of vehicles with obstacle avoidance.

- Optimise time and place of operations

- Coordination of smaller vehicles.

- Safe and efficient route planning.

- Precise retracing of previous paths, for example to ensure fertilizer being applied to seeded area.

- Drones for more accurate plant health analysis and process high-quality maps during every stage of crop growth.

- Efficient collection of data regarding livestock health as well as their location.

- Resource Optimization, through the use of wireless sensor networks and real time monitoring of the principal parameters in crop and livestock activities.

- Support in management of the harvest and post harvest processes as well as the traceability of agricultural products.

- Better yields and decreased production costs due to monitoring of variables that determine the behaviour of the crops. 

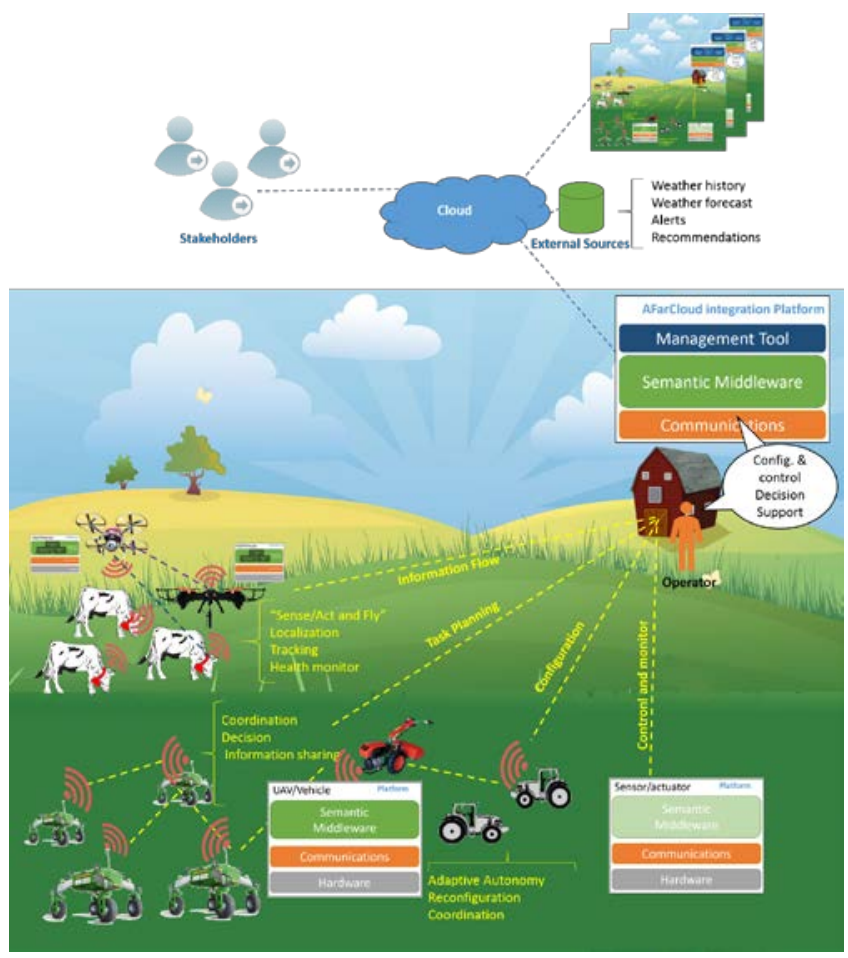

Fig. 1. AFarCloud overview

Fig. 1 illustrates a global view of the AFarCloud system. Measurements and other data from each farm will be collected in the cloud together with external data, for example weather history. Collecting and analysing data from several farms will give new business opportunities to farmers and other stakeholders in the food supply chain, such as . The semantic middleware will be distributed between vehicles and the management tool. Reliable communication is important as well as to select the correct level of autonomy for the different components in order to obtain robust solutions.

\section{VALIDATION SCENARIOS}

Thanks to the modular architecture approach, AFarCloud is intended to be applied in a plethora of farms with different facilities and requirements. In order to demonstrate the key functionalities of the project and perform a comprehensive validation procedure, a prototype of the solution will be deployed within three holistic demonstrators, including cropping and livestock management scenarios: Finland, Spain and Italy (in months 12, 24 and 35 of the project respectively). In addition, and following an incremental prototyping approach, AFarCloud will be validated also through a set of local demonstrators deployed in different locations (Latvia, Czech Republic, Italy, Spain and Sweden). These local demonstrators will be running during the lifetime of the project in order to test the specific set of functionalities for each location.

The location of the holistic and local demonstrators has been selected to have different climate and production areas across Europe. In order to validate the results of the project at the end of the project, baseline data will be gathered for each location in month 1 of the project. Thus, the validation procedure will compare the final figures obtained with the aforementioned baseline data. Detailed validation procedures will be developed in the project.
Following a cloud computing approach, provided by the AFarCloud project, these data will be available by a web dashboard. Therefore, it will be possible to gather all the data about farms among the various demonstrator sites; due to its remote access capability, it will be possible to create a knowledge base for the crop and livestock in Europe that contains all the information about the needs of the crop in different months of the years. This solution will offer a source of big data analysis for agricultural parameters, considering the opportunity of comparing the same cultivation at different latitudes and geographical sites.

AFarCloud will increase the whole farm productivity, gathering data to monitor the conditions of animals, crop and soil. It will be demonstrated on three holistic scenarios: cropping management, feed management, movement control, and animal's health monitoring, showing the flexibility of solutions for different crops and animals. The three holistic scenarios are a closed-loop system of systems, that combines cropping and livestock scenarios. They will include common technologies from the cropping management, such as selective monitoring, pests and illnesses detection and more targeted weed reduction, precision fertilization and harvesting optimization. AFarCloud technologies will produce maps of field status covering a range of parameters and make these available to the farmer. Fig. 2 depicts a conceptual diagram of the holistic scenarios based on an example for livestock nutrition management.

\section{Livestock Nutrition Management System}

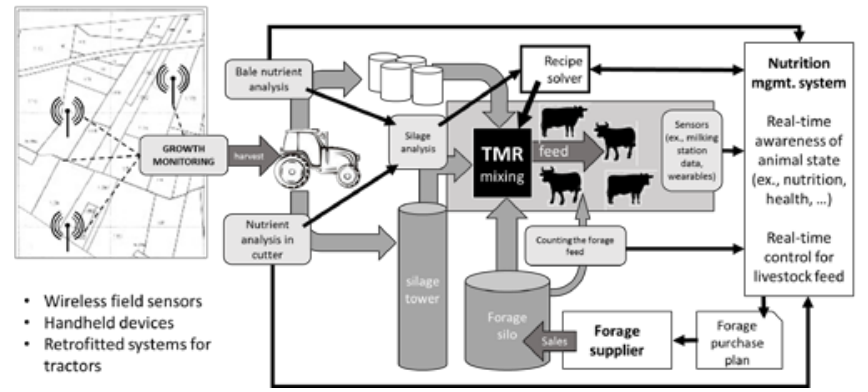

Fig. 2. Conceptual diagram of an AFarCloud holistic scenario.

In these scenarios, the objective of cropping is the generation of optimized feeder for livestock that has to be tamped before fermentation for proper silage. A livestock feed management system will be implemented to determine the amount of food to be distributed to the animals for their optimal nutrition and grazing management. Livestock movement and health will be also monitored through the use of wearables with sensors such as accelerometers, temperature sensor and GNSS. This data will be used to provide alerts concerning fertility, calving and nutritional habits among others.

Furthermore, AFarCloud demonstrators are a data mining infrastructure and actuators, while the main intelligence of farming - in terms of AFarCloud-is located in the cloud.

\section{A. AFarCloud cropping management scenarios}

AFarCloud cropping management scenarios (Fig. 3) will include a wide variety of species such as berries, fruits, grapes, cereals, and horticultural products in different locations across Europe. These scenarios will include, in addition to the crops themselves, the environment's meteorological conditions and the soil's condition, which are 
critical for understanding status of the fields and also to determine the measures that need to be taken.

Demonstration will show experimental sample of classification and reasoning system with capabilities of data gathering from heterogeneous sensor sources, data transport, and fusion with multilayer matrix of linked data to feed intelligence of AFarCloud based decision support system.

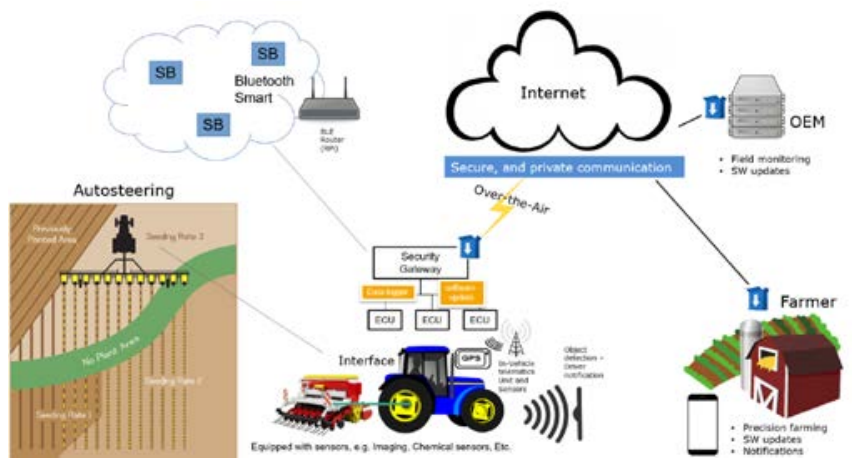

Fig. 3. Conceptual diagram of an AFarCloud holistic scenario.

From a technological point of view, implementation of sensors (whether WSN or sensors on board a UAV and/or ground vehicles) and actuator decision support, are key roles in all of the scenarios. In particular, overall system protection feature is able to follow plants at all the stages of their life (germination, growth, maturation, harvest, pruning needs etc.), protecting plants from the frost, weeds, pests and illnesses, which need to be detected at early stages and treated as soon as possible, and demonstrating both autonomous monitoring of the plant growth and management.

Autonomy, and autonomous systems, are central concepts in AFarCloud. Whereas monitoring of an area, a field or a barn, can be achieved similar to traditional automation, much like monitoring robot cells in an industrial setting, more advanced monitoring and manipulation missions require close collaboration between human operators and (semi)-autonomous systems. The key is adaptive autonomy, i.e. the ability for an autonomous system to adapt its level of autonomy to a given task or problem. Another important factor is information sharing between vehicles and machines, which will facilitate communication and collaboration. Thus, it is assumed that, an approach that assumes intelligent control by the operator through planning and continuous monitoring, adaptive autonomy for flexible plan execution, and extensive information sharing between all parts, will both increase the successful outcome of a mission plan, and support the operator when the mission plan needs revision in real-time.

AFarCloud framework will able to manage all these subscenarios with horticultural and vineyards demonstrators with their proper data in a unique integrated platform and knowledge base. The crop scenario has a common point: the real time monitoring of the crops and autonomous actuators via UAVs or specialized UGVs, as well as ad hoc IoT actuators equipment. The main features in crop demonstrator involve real time monitoring, doing action for the plants and analysis of data to achieve better results in the production. Drones and sensors-based with autonomous management, combined with other data sources (e.g. web weather forecasts, satellite imagery, etc.) are a good solution to have a fast, cost-efficient and exact analysis methods for nutrient balance in soil and vegetation. The different types of scenario in crop demonstrator show a real implementation of the concept of Systems of Systems where different CPS systems are interconnected in a framework being the core of AFarCloud platform. Vehicles and machines will be able to communicate with each other to share information and make real-time planning to address the mission goals defined by the farmer. Some particular innovative agronomic practices can help in better performance and reduced work in farmers. Data from sensors will be analysed in order to generate smart alerts and indicators, that will be fed to end users through intuitive multi-device interfaces, thus for instance helping to optimize the drip irrigation system already existing in the scenario or minimizing the amount of fertilizer released by a robot. All the modules (sensors and additional sources, data analytics and interfaces) will be integrated with the AFarCloud platform.

\section{B. AFarCloud livestock management scenarios}

Livestock management, through smart technologies, takes into account of three principal factors or issues with the essential concept of measurements about the different typical values needed to gather for that issue, and data analysis on the Cloud. In all the livestock farms taking part of the demonstrators, whether local or holistic, these are the factors that need a solution based on AFarCloud: feed management, health status and movement control. The conceptual overview is depicted in Fig. 4.

The livestock feed management can be improved by using a central planning system to determine the amount of food to be distributed to the animals for their optimal nutrition and grazing management tools. For example, the main food of cows is grass and it would be important to monitor the nutritional parameters of grass fed to the animals, but also to deploy wearables to monitor rumination.

The health status can be provided by sensors measuring vital parameters such as temperature, pulse, breathing, oxidereduction potential and $\mathrm{pH}$. In addition, behaviour monitoring is also relevant for detection of animal fertility or illness. Internet of Things networks such as SigFox, NB-IoT and LoRa can offer a breakthrough solution especially to the extensive livestock farmers. The use of long-range and low data-rate communications, and combing it with IMUs and GNSS technology can provide tracking information related to animal behaviour. The possibility of collecting data in the cloud allows unprecedented possibilities to carry out relevant statistical data analysis for efficient livestock management.

The third factor is the livestock movement control, the daily livestock operation is based on the management of the animals that are grazing. For example, in those farms with large extensions of pastures, it is difficult to find the cows and calves. It is necessary to know the location of the cows, those that close to the calving date, those that in-heat, the position of their calf, the health of cows and calves. 


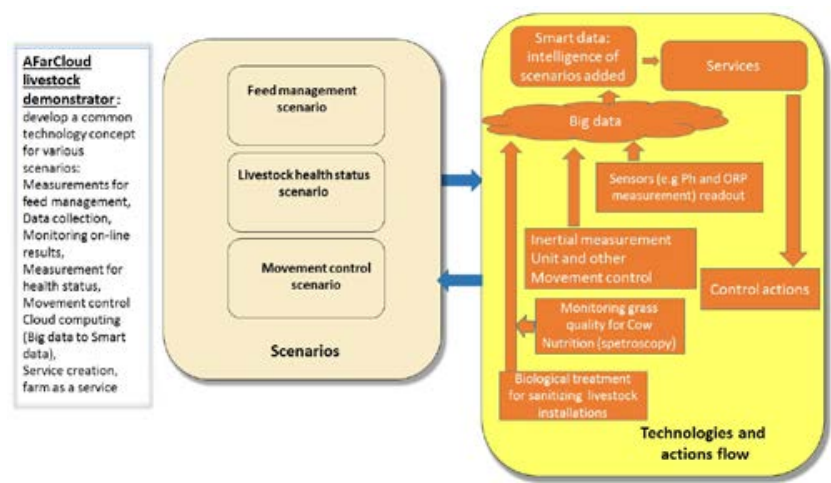

Fig. 4. AFarCloud livestock demonstrator overview.

\section{IMPLEMENTATION}

\section{A. AFarCloud work packages}

The AFarCloud project will last for 36 months. The work that will be carried out is divided into eight (8) Work Packages (WPs). The work inside the WPs that design and develop (WP2-WP6), as well as demonstrate and validate (WP7) these technologies will follow the incremental prototyping methodology, and thus generate several sets of outcomes (Fig. 5).

Thus, the structure, contents, objectives and their interdependencies are as follows.

Project Management (WP1) aims to monitor and coordinate all project activities and ensure conformance with the project plan and quality plan, including tasks to coordinate efforts among the members of the project towards common objectives in the different work packages, track the progress of the project by means of scheduled meetings that will take into account the expected results according to the milestones and obtain outputs that are conveniently integrated in the project as holistic, uniform contributions instead of dispersed pieces of work.

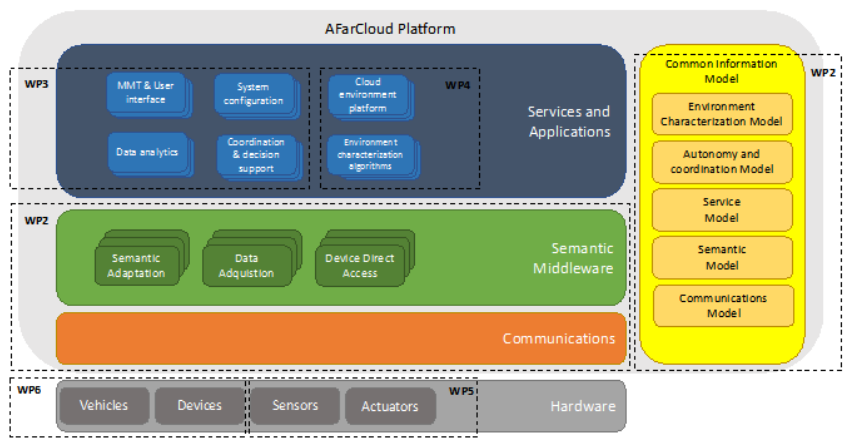

Fig. 5. AFarCloud work packages.

Research and Innovation Technological design and development activities are concentrated to WP2 - WP6. In WP2 - System Requirements, Architecture Specification and Implementation, user and system requirements of the AFarCloud platform will be idenfied. In addition, the semantic middleware and a secure communication system will be implemented. Thus, the WP2 is central in that it will identify user needs and requirements, which is the starting point of the WP3-WP6 work, and also be the host for the main infrastructure. The users' perspectives and the services and applications needed to utilize the AFarCloud solution is designed and developed in WP3 - Intelligent Coordination and Decision-support Solutions for Autonomous Operations, more specifically a Mission management tool for the user to access the whole system, make highlevel and task plans for future missions, configure and coordinate of (semi)-autonomous systems, and legacy systems, and also a decision-support system for in-depth analysis of collected data, and making decision. The data that is visualised and used for different actions will be collected and processed through the solutions in the WP4 Environment Characterization Platform. Thus, this WP assumes a solution for combining the information from the data streams provided by the sensors, and data available through cloud, through big data analytics.

One essential aspect of the AFarCloud project is the development and integration of the autonomous systems and the legacy systems into the AFarCloud platform in order to provide a wide spectrum of solutions for different categories of end users. This core of this critical work will be done in the WP6 - Autonomous System Development and Legacy System Integration. In WP5 - Sensor and Actuator Development, the focus is on the design and developing of the necessary sensors and actuators to perform monitoring, sampling and actuation over different plants and animals. These sensors can be part of autonomous systems and legacy systems, as well as the rest of the infrastructure.

Demonstration \& Prototyping activities will be organized in the WP7 - Demonstrators definition, integration, verification and validation, which tackles with the development and configuration of the infrastructure for carrying out the integrated demonstration and validation of the overall AFarCloud objectives. Finally, WP8 Dissemination, Exploitation and Standardization aims at increasing the innovation capabilities of the AFarCloud partners, such as training activities in building value networks, customer value creation and the evaluation of business impact for farming companies through a comparison with data. This WP has also the aim of expanding the State-of- the-Art in the fields of knowledge related with the project by publishing research articles in well- established scientific journals, as well as contributing in congresses and events of interest for the consortium and exploiting the results obtained in the project.

\section{B. Consortium as a whole}

AFarCloud consortium is comprised of $\mathbf{5 9}$ organizations from 13 European countries (Fig. 6), and it includes a well-balanced mix of industrial companies (large and SMEs), as well as academic partners (research organisations and universities):

- 9 large industrial companies: Acciona and Bosonit (Spain), NXP e Intrasoft (Belgium), TTControl, AVL List, AVL-CD and AMS (AT), and STMicroelectronics (Italy)

- 26 Small and Medium-sized Enterprises (SMEs): TST, EncoreLab, Rovimatica, Sensowave, Hi-Iberia and Carrera d'en Bas (Spain), Nuromedia, Germandrones and Logic Way (Germany), PDMFC (Portugal), Maritime Robotics (Norway), Imagimob, Spacemetric, Qamcom and Senseair (Sweden), MTech, Probot and PehuTec (Finland), BetterSolutions (Poland), IMA and Lesprojekt (Czech 
Republic), KES, RoTechnology, Archa, and ESTE (Italy), and EXODUS (Greece).

- 10 research organisations: Tecnalia (Spain), AIT (Austria), Instituto de Telecomunicações Aveiro (Portugal), SINTEF (Norway), RISE-SICS and RISEACREO (Sweden), VTT and Centria (Finland), Institute of Animal Science (Czech Republic), and CNR (Italy)

- 14 universities: Universidad Politecnica de Madrid (Spain), Technische Universität Dresden and Saarland University (Germany), Katholic University of Leuven (Belgium), Vienna University of Technology (Austria), Mälardalen University (Sweden), Gdansk University of Technology (Poland), University of West Bohemia and Charles University (Czech Republic), Universitá degli Studi di Parma, Universitá degli Studi del Sannio and University of L'Aquila (Italy), IMCS (Latvia), and Harokopio University of Athens (Greece).

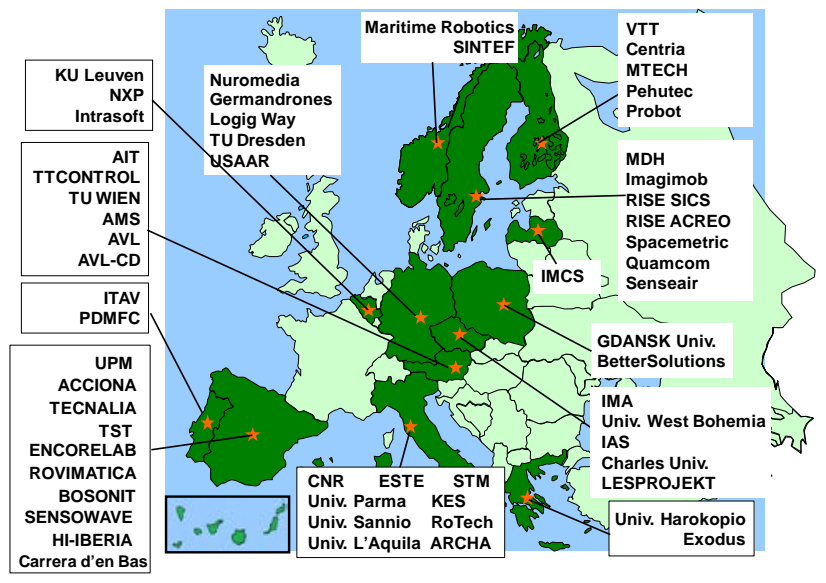

Fig. 6. AFarCloud consortium's geographical balance.

The AFarCloud Consortium is a well-balanced team that contains leading European players, combining business know-how and deep technical insights. This ensures that the partners are well positioned to cooperate and collaborate to respond to the global technological challenge. Highly skilled AFarCloud partners each of them contributing with their specific expertise and know-how to the joint research and development activities will drive improvement of scientific and technological cooperation for mutual benefit.

This consortium has the right balance between academia and industry in order to achieve cutting-edge research results in the field of Electronic Components and Systems (cyber physical systems, sensor and robotics development and integration, autonomous systems) applied to the Smart Farming domain. On the academic side, there are some of the more important European universities and research institutions in the fields of embedded systems, robotics, smart farming and agriculture research. Their competences are aligned with the technical interest and needs for successfully carrying out the project. This set of academic partners will contribute stereo vision algorithms and computer vision techniques, real time pattern recognition algorithms; wireless networked sensor systems; knowledge in semantic interoperability of devices, including resource limited devices and robot cooperation; on board decisional architectures for farming autonomous vehicles; autonomy deployment for task planning, task supervision and fault management; positioning, identification and location technologies; technologies for robot's navigation system, route planning, object detection, tracking; precise technologies for localization of objects; cloud architectures and big data analytics.

To ensure the commercial exploitation of these excellent competences strong industrial partners with a background in farming technology and/or communications sector drive the consortium. The large industrial companies and SMEs involved in the project cover the main industry needs for AFarCloud with key profiles in manufacturing of autonomous systems (UAVs, terrestrial vehicles and robots); competences on automation, control, robotics and actuators for farming applications; on vision solutions for the imaging and navigation systems; on secure network communications; on sensor data gathering systems; on software engineering for designing and testing industrial products via virtual experience for a high level Systems Of Systems (SoS) view, accurate geometry of scenes. Finally, the consortium is completed with industrial partners who define the AFarCloud use cases ("voice of the customer") and provide farming testing facilities platforms that allow demonstrating, validating and verifying the AFarCloud approach and technologies.

In the other hand, the AFarCloud Consortium is built-up by a perfect mix between experienced partners already participating in EU projects of the ECSEL programme, and newcomers with very valuable skills in the Smart Farming application domain and in the technologies that will be applied to AFarCloud.

\section{CONCLUSIONS}

This paper has presented the AFarCloud ECSEL JU project. It will provide a distributed platform for autonomous farming that will allow the integration and cooperation of agriculture Cyber Physical Systems in real-time in order to increase efficiency, productivity, animal health, food quality and reduce farm labour costs. As the paper is written after the first months of the project, it concentrates rather on the project introduction while project results will be presented later.

\section{ACKNOWLEDGMENTS}

A special thanks to all the AFarCloud consortium people that have worked on the AFarCloud proposal on which this paper is based on. The AFarCloud project is funded from the ECSEL Joint Undertaking under grant agreement $\mathrm{n}^{\circ} 783221$, and from National funding.

\section{REFERENCES}

[1] Gnip, P., Charvat, K., \& Krocan, M. (2008). Analysis of external drivers for agriculture. In World conference on agricultural information and IT, IAALD AFITA WCCA 2008, Tokyo University of Agriculture, Tokyo, Japan, 24-27 August, 2008 (pp. 797-801). Tokyo University of Agriculture.

[2] Vitousek, P. M., Mooney, H. A., Lubchenco, J. \& Melillo, J. M. Human domination of earth's ecosystems. Science 277, 494-499 (1997)

[3] Carpenter, S. R. et al. Nonpoint pollution of surface waters with phosphorus and nitrogen. Ecol. Applic. 8, 559-568 (1998)

[4] http://cordis.europa.eu/project/rcn/73844_en.html

[5] http://sitem.herts.ac.uk/aeru/footprint/ 